\title{
The role of the radiation oncologist in oncology today
}

\author{
Ferran Guedea
}

$\mathrm{O}^{\prime}$ ncology is the medical knowledge that drives and determines the treatment of patients with cancer. In Spain, various oncological specialties are recognised and these have co-existed for decades, although the specialty with the longest tradition is probably radiation oncology. While all oncological specialties have their own specific content, they also share a common knowledge base. As a result, specialists in the different areas of oncology all have something unique to contribute to our understanding of the disease; together, these specialists provide a comprehensive model of patient-centred care [1-5].

Approximately $60 \%$ of cancer patients will be treated with radiotherapy at some point in the course of their disease. Consequently, it is clear that radiation oncology is an essential part of oncology today, an observation that is further supported by the multidisciplinary approach exemplified by tumour boards and multidisciplinary treatment units. This team-based, comprehensive model is perhaps best illustrated by the consensus treatment decisions made by tumour boards, an approach which was recently validated at a conference entitled "Multidisciplinary Cancer Care as a Model for Quality Care", which led to the signing of the "Institutional Declaration for the Development of Multidisciplinary Cancer Care in Spain" at the Ministry of Health on 27 May 2010, recently published in this journal [6]. Two important conclusions were reached at this conference: (1) we must make implementation of this strategy in our hospitals a high priority and (2) the opinion of all specialists involved in the therapeutic decision should be given equal weight-in other words, no single medical specialty should have a monopoly on cancer care.

The wellbeing of the cancer patient cannot be guaranteed by a "star performer". Quality care depends on a team of professionals who know how and when they need to act, and who are perfectly willing to work collaboratively by contributing their specialised knowledge and skills to

\section{F. Guedea (ه)}

Spanish Society of Radiation Oncology (SEOR)

Catalan Institute of Oncology (ICO)

University of Barcelona

Av. Gran Via, s/n - km, 2,7

ES-08907 L'Hospitalet de Llobregat, Barcelona, Spain

e-mail: guedea@iconcologia.net a common project. The true professional knows that he or she will be required to take on a leading role at times while accepting a more discreet, secondary role (though not for that any less necessary or effective) at other times [7, 8].

Health-care administrators often desire to know how the various cancer therapies impact patient survival. The Swedish Council on Technology Assessment in Health Care addressed this question more than 10 years ago $[3,4]$. This group found that, of patients whose cancer was cured, an estimated $49 \%$ were cured by surgery alone, $40 \%$ by radiotherapy alone or in combination with other therapeutic modalities, and $11 \%$ by chemotherapy alone or combined with other therapies. In recent years, the trend has been to use (concomitantly in most cases) combined radiotherapy and chemotherapy (or new targeted drugs) to improve locoregional disease control and survival. We should keep in mind, however, that chemoradiotherapy improves overall results via two primary mechanisms: spatial cooperation (thanks to the early activity of chemotherapy on subclinical metastatic disease) and also through the radiopotentiating/ radiosensitising effect of some drugs (which are only effective when administered co-adjuvantly with the primary physical treatment). Combined chemoradiotherapy also makes it possible to increase the organ conservation rate achieved by radiotherapy.

Oncology today is characterised by revolutionary advances in technology (thanks to continuing progress in computer science) and pharmacology (thanks to advances in molecular biology). Given these circumstances, it behooves us as professionals to reflect on the future of radiation oncology and our role in the comprehensive management of cancer patients. Improvements in radiotherapeutic techniques have been nothing short of spectacular over the last decade, although this progress has come at the expense of ever-increasing complexity and time requirements (IMRT, IGRT, SBRT, etc.). Not only have volume delimitation, planning and dosimetry all become more time consuming and laborious, but quality control issues have been added to our daily work. Unfortunately, the important technical component of our field limits the time we have available to dedicate to the clinical (and biological) aspects of treatment.

It is the author's opinion that we need to learn to delegate part of our patient care workload to other profession- 
als (physicists, nurses, radiotherapy technologists, imaging technicians, quality control technicians) so that we can focus more on the clinical aspects of care. Fortunately, intelligent planning systems that automatically recognise anatomical structures are starting to emerge, a development that implies the possibility (theoretical at the moment) of automatic contouring of anatomical volumes. All the progress that has been made in recent years-advances in diagnostic imaging (CT, MRI, PET), proliferation and hypoxia studies, advances in planning systems (image fusion, inverse planning), a better understanding of organ radiotolerance and the aforementioned potential for automatic contouring of volumescould, in the near future, reduce or even eliminate the need for a specialisation centred exclusively on radiotherapy. As Corn and Glatstein put it: "A problem is our seduction by technology. The new toys have captured our collective fancy." A statement that is seconded by Zietman [2, 9].

We would do well to remember also that basic (or translational) research, clinical investigation and training are also essential for the development of a medical specialty. Indeed, the SEOR has made a concentrated effort to support continuing education programmes, in part by creating a very successful teaching platform (EEOR-Spanish School of Radiation Oncology). Clinical research is important because it promotes an environment of scientific excellence and quality while translational investigation obliges radiation oncologists to become more deeply involved in the field. It seems clear that translational research is essential to ensure the future viability of our specialty.

These days, there is much talk about personalised cancer treatment and I believe that the radiotherapeutic treatment of cancer is one of the clearest examples of that concept: treatments can be adapted to the clinical-pathological and prognostic characteristics of the tumour. Moreover, other factors, such as the areas of expertise of each centre, the expected quality of life of the patient and psychosocial aspects of treatment, can also be considered in the decisionmaking process. This means, however, that the information given to the patient must, of necessity, be highly complex and require careful consideration. For these reasons, it is imperative that the patient receive impartial advice that is not biased in any way.

What is the author's opinion regarding the role of the radiation oncologist in oncology today? The following is a summary of the characteristics that a highly qualified specialist in radiation oncology should possess. He or she should:
1. be a good clinician who is well-versed in all relevant clinical aspects of the disease of cancer;

2. be thoroughly familiar with the technical aspects of radiotherapy, as well as with emerging technologies and their side effects;

3. have a good understanding of how and when to prescribe medications and molecular therapies for cancer patients, and the side effects of these therapies;

4. be fully aware of the fundamental role of clinical research to achieve progress in oncology, particularly with regards to evidence-based medicine and clinical trials;

5. be aware of the importance of translational research in the development of our specialty; and support the transfer of such research from the laboratory bench to the clinic;

6. have psychological empathy for the patient, and be aware of all the important psychosocial aspects that can affect cancer patients;

7. possess a solid belief in multidisciplinary decisionmaking for cancer patients and for participatory involvement in comprehensive, patient-centred care;

8. be aware of the importance of disseminating the scientific aspects of our specialty (publications, congresses, etc.);

9. be involved in ongoing training and university teaching;

10. be open to collaboration with other national and international centres as well as European (especially ESTRO) and Latin American (in particular ALATRO) scientific societies. Spain is a bridge between Europe and Latin America, but must continue to strengthen that role.

It is not my intention to categorically assert that all radiation oncology specialists must be experts in all the areas listed above. There will inevitably be professionals who focus more on the technical aspects of radiotherapy, while others concentrate on the association between drugs and radiotherapy or on clinical or translational research. In fact, this is the case today: most radiation oncologists specialise in one or several tumour localisations (prostate, breast, lung, brain, etc.), even though they all adhere to and fully understand the fundamental precepts of radiotherapy.

In conclusion, we radiation oncologists should recognise the need to accept these proposals and apply them in our daily practice of patient care. By doing so, we can continue to strengthen the scientific prestige and recognition that our specialty deserves. Moreover, adherence to these ideas will allow us to continue providing patients with optimal therapeutic care.

\section{References}

1. (2006) Orden SCO/3142/2006, de 20 de septiembre, por la que se aprueba y publica el programa formativo de la especialidad de Oncología Radioterápica. Boletín Oficial del Estado 245, 13 October, 35519-35527

2. Zietman A (2008) The future of radiation oncology: the evolution, diversification and survival of the specialty. Semin Radiat Oncol 18:207-213

3. (1996) SBU-The Swedish Council on Technol- ogy Assessment in Health Care. Radiotherapy for Cancer. Volume 1. Acta Oncol 35[Suppl 6]

4. (1996) SBU-The Swedish Council on Technology Assessment in Health Care. Radiotherapy for Cancer. Volume 2: a critical review of the literature. Acta Oncol 35[Suppl 6]

5. Gouveia J, Coleman MP, Haward R et al (2008) Improving cancer control in the European Union: conclusion from the Lisbon roundtable under the Portuguese EU presidency, 2007. Eur J Cancer 44:1457

6. SEOQ, FESEO, SEP et al. (2010) Institutional
Declaration for the Development of Multidisciplinary Cancer Care in Spain. Clin Transl Oncol 12:587-589.

7. Maciá M (2007) Rebelarse, Someterse o Pactar. SEOR Actúa 28

8. Meropol NJ, Schrag D, Smith TJ et al (2009) American Society of Clinical Oncology guidance statement: the cost of cancer care. J Clin Oncol 27:3868-3874

9. Corn B, Glatstein E (2009) Editorial: the most important question. Int J Radiat Oncol Biol Phys 74:985-986 